TITLE:

\title{
Conductance of atom-sized contacts of transition metals at room temperature
}

$\operatorname{AUTHOR}(S)$ :

Moriguchi, Y.; Yamauchi, K.; Kurokawa, S.; Sakai, A.

CITATION:

Moriguchi, Y....[et al]. Conductance of atom-sized contacts of transition metals at room temperature. Surface Science 2012, 606(11-12): 928-932

ISSUE DATE:

2012-06

URL:

http://hdl.handle.net/2433/155099

RIGHT:

C 2012 Elsevier B.V.; この論文は出版社版でありません。引用の際には 出版社版をご確認ご利用ください。; This is not the published version. Please cite only the published version. 


\title{
Conductance of atom-sized contacts of transition metals at room temperature
}

\author{
Y. Moriguchi, K. Yamauchi, S. Kurokawa, A. Sakai* \\ Department of Materials Science and Engineering, Kyoto University, Sakyo-ku, Kyoto \\ 606-8501, Japan
}

\begin{abstract}
Exploiting the mechanically controllable break junction technique, we have measured the conductance of atom-sized contacts of $\mathrm{Fe}, \mathrm{Co}$, and $\mathrm{Ni}$ at room temperature under ultrahigh vacuum conditions. The conductance histogram of $\mathrm{Fe}$ exhibits a broad peak around $2.5 G_{0}\left(G_{0} \equiv 2 e^{2} / h\right)$, whereas those of Co and Ni show no conductance peaks. However, the histograms of Co and Ni display different structures: While the Co histogram is simply flat, the Ni histogram reveals an appreciable background. Our experimental results are compared with previous results obtained at cryogenic and room temperatures, and the observed peak missing in our room-temperature histograms of $\mathrm{Co}$ and $\mathrm{Ni}$ is discussed. Keywords: Atom-sized contacts, Conductance, Iron, Cobalt, Nickel
\end{abstract}

\footnotetext{
${ }^{*}$ Corresponding author

Email address: sakai-akira@z06.mbox.media.kyoto-u.ac.jp (A. Sakai)
} 


\section{Introduction}

Electron transport through atom-sized metal contacts has been a subject of intensive theoretical and experimental studies in the past decade [1]. Extensive data has been collected on the conductance of atom-sized contacts of typical metals such as $\mathrm{Au}, \mathrm{Ag}, \mathrm{Cu}$, and $\mathrm{Al}$, particularly their single-atom contacts (SACs). Atom-sized contacts of the $3 d$ transition metals $\mathrm{Fe}$, Co, and $\mathrm{Ni}$ have also attracted much attention because of the possible manifestation of spin polarization effects on their conductance. When a ferromagnetic contact has transparent conduction channels, the polarization of the valence electrons lifts up the spin degeneracy of each channel and yields a conductance that changes in units of $G_{0} / 2$, instead of $G_{0}$, as in ordinary conductance quantization $\left(G_{0} \equiv 2 e^{2} / h\right.$ is the conductance quantum unit). A number of experimental studies have been carried out in search of such fractional conductance quantization in ferromagnetic atom-sized contacts, and both positive $[2,3,4,5,6,7,8]$ and negative $[9,10,11,12,13,14,15,16,17,18,19]$ results have been claimed. In positive experiments, sharp peaks were observed at $n G_{0} / 2$ in the conductance histogram, except in one experiment on Fe [3] that found conductance peaks at $n G_{0}$. In contrast, all negative experiments unanimously reported a broad single peak located between $1 G_{0}$ and $2 G_{0}$, which was consistent with the theoretical conductance, in which contributions of the partially transparent conductance channels of $3 d$ electrons sum up to yield varied conductance values.

The above discrepancy concerning the conductance of atom-sized Fe, Co, and $\mathrm{Ni}$ contacts has often been attributed to differences in the measurement method and environment used in each experiment. Indeed, experiments have been performed under diverse conditions, at temperatures ranging from cryogenic temperatures to above $1000 \mathrm{~K}$ and in environments ranging from ultrahigh vacuum to aqueous solutions. With the notable exception of one STM experiment [5], most previous experiments [12, 13, 14, 16, 17, 18, 19] conducted under cryogenic vacuum conditions at $4 \mathrm{~K}$ or below have employed the mechanically controllable break junction (MCBJ) technique for producing atom-sized con- 
tacts and consistently observed a broad conductance peak in the conductance histogram [20]. Experimental methods and environments are more diversified at room temperature than at $4 \mathrm{~K}$. For example, atom-sized contacts have been produced by different kinds of break junctions, such as wire-wire [2, 3], wirefilm [4], relays [11], electrodeposited nanowires [6], and STM [7, 8, 10, 16, 15]. Experimental environments have also ranged from ambient air $[4,3,10,11]$ to high vacuum [2, 7], paraffin oil [8], and electrochemical solutions [6, 15, 16]. Some studies reported $n G_{0} / 2$ peaks while others found a broad single peak in agreement with low-temperature MCBJ experiments. This wide variety of methods and environments employed in previous experiments is most likely the main cause of discrepancies between the reported conductance data. Thus, a proper investigation of the conductance of atom-sized Fe, Co, and Ni contacts at room temperature requires conducting experiments using a "standard" method and environment.

As mentioned above, all previous MCBJ experiments conducted in cryogenic vacuum have yielded consistent results for $\mathrm{Fe}, \mathrm{Co}$, and $\mathrm{Ni}$ atom-sized contacts, and the conductance data produced can conveniently be regarded as a reference. Thus, a natural choice for the "standard" method and environment at room temperature would be MCBJ and ultrahigh vacuum (UHV), respectively. For Fe, Co, and Ni contacts, however, no experiments have yet been carried out with an UHV-MCBJ at room temperature. To fill this knowledge gap and investigate the room-temperature conductance of $\mathrm{Fe}$, Co, and $\mathrm{Ni}$ atom-sized contacts properly, we have, in the present study, made UHV-MCBJ measurements of the conductance of these metals. Comparison of our results with the low-temperature reference data and with results obtained for solutions [16] reveals how the temperature and solution environment affect the conductance histograms of Fe, Co, and Ni atom-sized contacts, as we will discuss in Sec. 3.

\section{Experiment}

The UHV-MCBJ used in the present experiment is the same as that employed in our previous study on the break voltage of single-atom contacts, and 
its details have been described elsewhere [21]. Samples are 0.1-0.25 mm diameter wires of 99.99-99.999\% purity. First, a piece of thin wire is fixed with a vacuum sealant onto a bending substrate (UBE UPISEL-C) at two adjacent points. After notching at the center of the two fixed points, the wire is broken at the notch by bending the substrate in UHV using a mechanical linear-motion feedthrough. The two ends of the broken wire form a pair of electrodes, the gap between which can be fine-tuned by changing the amount of substrate bending using a piezo actuator. Through repeated extension and retraction of the piezo actuator, we opened and closed the junction.

In MCBJ, the displacement of electrodes is reduced relative to that of the piezo actuator by a factor that depends on the geometry of each MCBJ specimen, specifically on the separation $u$ between the two fixed points of the metal wire [1]. If we assume $u \sim 0.2 \mathrm{~mm}$, the opening/closing speed of the junction calculated from the reduction ratio and the extension/retraction speed of the piezo actuator becomes $\sim 0.7 \mathrm{pm} / \mathrm{s}$. However, the actual junction speed varies from specimen to the specimen because the wire fixing was carried out manually, and thus the separation $u$ could not be determined precisely. To compensate for this variation, we made slight adjustments to the stroke of the piezo actuator so that the last stage of the conductance falloff from $5 G_{0}$, which will be discussed below, could be captured in the same time window. Though this procedure is not an accurate speed calibration, we believe that it reduces the variation in junction speed and helps to equalize the junction speed across specimens.

We recorded the temporal evolution of the junction conductance (referred to as a "conductance trace") during junction opening. The conductance decreases with time, reflecting the reduction in junction size induced by necking deformation. Shortly before contact failure, however, the conductance often changes stepwise and exhibits several plateaus. These conductance plateaus correspond to certain stable contact geometries, and those plateaus that appear in the last stage of the junction breakup are due to atom-sized contacts. However, because of the lack of reproducibility in nano-scale contact necking, every junction opening yields different conductance traces. Thus, we need to ana- 
lyze the conductance of atom-sized contacts statistically by acquiring a large number of traces and organizing them into a conductance histogram. Stable atom-sized contacts usually yield long, reproducible plateaus, and hence produce well-defined peaks in the histogram. The first peak is typically attributed to the single-atom contact.

In the present experiment, we measured the conductance trace under a constant bias of $100 \mathrm{mV}$. The conductance histogram of Fe was constructed from 7,300 traces in total measured on 5 specimens. The Co and Ni histograms represent, respectively, 3,000 traces on 3 specimens and 5,100 traces on 4 specimens. All measurements were made at room temperature under a vacuum below $2 \times 10^{-8} \mathrm{~Pa}$.

\section{Results and discussion}

\subsection{Conductance traces}

Figure 1 presents examples of conductance traces observed on Fe, Co and Ni breaking junctions. We note that all these traces have distinct features, each showing several plateaus. Most of the traces, however, decrease monotonically and exhibit no visible plateaus. As mentioned before, the breaking speed of the junction is $\sim 0.7 \mathrm{pm} / \mathrm{s}$, which is much lower than the typical speed used in MCBJ experiments. As demonstrated by Tsutsui et al. [22], under such slow breaking speeds, junctions are likely to undergo self-breaking, during which the junction lifetime would be lengthened owing to the relaxation of tensile stress within the junction. Even with this life-prolonging effect, however, the observed plateaus are still short-lived, lasting well less than $1 \mathrm{~s}$. This is in marked contrast to the break of Au junctions, single-atom contacts of which show a lifetime longer than $10 \mathrm{~s}$ at $0.8 \mathrm{pm} / \mathrm{s}$ at room temperature [22]. As noted in the previous section, the plateau states generally correspond to contact geometries that are comparatively stable, but the plateau states of $\mathrm{Fe}$, Co and Ni are very shortlived and far less stable at room temperature than those of noble metals. Their stabilization requires some extrinsic mechanisms, as we will discuss in Sec. 3.5. 


\subsection{Conductance histogram: $\mathrm{Fe}$}

Figure 2(a) shows the conductance histogram of Fe obtained at room temperature in UHV. The histogram reveals a single broad peak, the maximum of which is located around $2.5 G_{0}$. The peak appears asymmetric, with an appreciable tail on its higher conductance side that extends beyond $3 G_{0}$. Previous MCBJ experiments on Fe at $4 \mathrm{~K}[12,13]$ reveal a single peak at $\sim 2 G_{0}$, considered to represent the single-atom conductance of Fe. The conductance peak reported by Ludoph and van Ruitenbeek [12] is cusp-shaped and narrowly located at $2.2 G_{0}$ with an apparent FWHM of $\sim 1 G_{0}$. Compared to their sharp peak at $4 \mathrm{~K}$, our room-temperature peak appears significantly broader, mainly owing to the high-conductance tail. Because there is no such tail at $4 \mathrm{~K}$, the tail observed at room temperature is likely a thermal effect. At room temperature, a contact can follow any one of a more diversified set of deformation paths during junction break than are available at $4 \mathrm{~K}$, and thus it can form more varied atomic arrangements before shrinking to a single-atom contact. In fact, the conductance trace shown in Fig. 1(a) exhibits several plateaus that appear above the peak conductance $2.5 G_{0}$. Such contact geometries with a conductance above $2.5 G_{0}$ would form more abundantly at room temperature than at $4 \mathrm{~K}$ and contribute to the rise in intensity on the higher conductance side of the peak. The histogram, however, exhibits no similar intensity increase on the lower conductance side of the peak and forms a relatively sharp peak edge at $\sim 2 G_{0}$. This suggests that the lowest conductance of atom-sized Fe contacts, i.e., the singleatom conductance of $\mathrm{Fe}$, would be $\sim 2 G_{0}$. This result for room temperature is in good agreement with the low-temperature reference value, $2.2 G_{0}$ [12]. Thus, our experimental results show that the conductance histogram of Fe, with its single-atom peak at $\sim 2 G_{0}$, remains essentially unchanged as the temperature rises from 4 to $300 \mathrm{~K}$. Room temperature broadens the single-atom peak significantly on the higher conductance side, but not so much as to smear it out. Our Fe histogram also shows none of the integer quantized peaks reported in a previous experiment conducted at room temperature [3], suggesting that the $n G_{0}$ peaks are unlikely to be an intrinsic feature of atom-sized Fe contacts. 


\subsection{Conductance histogram: Co}

In contrast to $\mathrm{Fe}$, the conductance histogram of atom-sized Co contacts (see Fig. 2(b)) is entirely flat, exhibiting no peaks. A couple of plateaus can be observed in the conductance traces shown in Fig. 1(b), but these do not appear at fixed positions and yield no conductance peaks in the histogram. A partial histogram constructed from the data on one specimen shows a very broad bump around $1 G_{0}$, but the total histogram retains no such features, showing an entirely smooth distribution. Clearly, there exist no preferred conductance states for Co break junctions at room temperature.

Our histogram agrees with the featureless histogram obtained by Hansen et al. [11] for Co relay contacts but disagrees with the histograms obtained at $4 \mathrm{~K}$ or below $[13,17,18,19]$, which consistently exhibit a well-defined single-atom peak at $1.3 G_{0}$. It is not yet fully understood why the low-temperature Co peak disappears at room temperature. We note similar cases of peaks going missing at room temperature in metals other than Co. The conductance histogram of Zn, for example, exhibits a single-atom peak at $4 \mathrm{~K}$ [23], but none at room temperature [24]. The same behavior is observed for the single-atom peaks of $\mathrm{Mg}$ [25] and $\mathrm{Ga}$ [26] as well. A common feature of these metals is that they all have a non-cubic crystal structure, with $\mathrm{Co}, \mathrm{Zn}$, and $\mathrm{Mg}$ being HCP metals. In the case of Ga, complex deformation processes of the Ga crystals are considered to be a possible cause of the missing peak at room temperature [26]. We also found that the room-temperature conductance histogram of ductile Mg alloys preserves several low-conductance peaks that cannot be observed in the case of pure Mg. [27] These results suggest that the deformation characteristics of these metals, presumably the small number of active slip systems in non-cubic crystals, might be responsible for the single-atom peak going missing at room temperature. However, we note that some HCP metals exhibit peak structures at $>3 G_{0}$ in their room-temperature histograms so that the HCP structure alone cannot fully account for the complete flatness of the Co histogram.

Another mechanism that tends to flatten the conductance histogram at room temperature is the thermal broadening of histogram features, as we will discuss 
with regard to the Ni histogram in the next section. However, the Co histogram has a different shape from $\mathrm{Ni}$ histograms, resembling more the typical histograms of brittle junctions. This implies that in the case of Co and Ni contacts, thermal fluctuations in their contact configuration have different effects on their conductance. Thus, if any thermal peak smearing occurs, it would be element-specific and more complicated than simple peak broadening. This whole discussion clearly indicates that the key issue is to identify the contact geometries produced during the breaking of Co nanocontacts and determine their conductance. The relationship between contact geometry and conductance has already been theoretically investigated for some FCC metals [28] including Ni, using molecular dynamics simulations combined with conductance calculations. Similar detailed studies on contact evolution are critically needed for Co and other HCP metals for elucidating their peculiar room-temperature conductance histograms.

\subsection{Conductance histogram: Ni}

Different from $\mathrm{BCC}$ Fe and $\mathrm{HCP} \mathrm{Co}, \mathrm{Ni}$ is a typical FCC metal like $\mathrm{Au}$, $\mathrm{Ag}, \mathrm{Cu}$ and $\mathrm{Al}$. Because these noble metals and $\mathrm{Al}$ all exhibit a single-atom conductance peak at both low temperature and room temperature, the same can be expected for Ni. Specifically, a broad peak at $\sim 1.3 G_{0}$, commonly reported in previous experiments $[13,14]$ at $4 \mathrm{~K}$, is expected to appear at room temperature. Contrary to this expectation, however, the room-temperature histogram of $\mathrm{Ni}$ depicted in 2(c) only shows a broad background, with no distinct peaks. Small bumps can be recognized around $1.3 G_{0}, 2.5 G_{0}$ and $5 G_{0}$, but these are too obscure to be identified as well-defined conductance peaks. Thus, as in the case of $\mathrm{Co}$, the conductance peaks of $\mathrm{Ni}$ are missing at room temperature. However, there is a marked difference between the $\mathrm{Ni}$ and Co histograms: While the Co histogram is simply flat, the Ni histogram exhibits an appreciable background. This background comes from conductance traces such as the one shown in Fig. $1(\mathrm{c})$, where the conductance decreases rather smoothly without any discrete steps or plateaus. Occasionally, several plateaus appear as shown in Fig. 1(d) 
(in this trace, a plateau happens to appear around $1.3 G_{0}$ ), but these plateaus do not occur at fixed positions and can hardly produce well-defined peaks in the histogram.

We first compare our Ni histogram with those obtained in previous experiments at room temperature. As mentioned in Sec. 1, previous roomtemperature Ni histograms are significantly varied, from featureless histograms [10] to those showing sharp half-integer conductance peaks. $[2,4,6,7,8]$ Our histogram clearly agrees with the former, indicating that the fractional conductance peaks observed in some experiments may not be an intrinsic property of atomsized Ni contacts. We note that one previous histogram [7] obtained under high vacuum (and at high temperatures) also lacks clear peak features. Fractional peaks are thus likely due to certain environmental effects.

Our room-temperature $\mathrm{Ni}$ histogram does not reproduce the $1.3 G_{0}$ peak commonly observed at $4 \mathrm{~K}$. We have at present no definite explanation for why this $1.3 G_{0}$ peak is missing at room temperature. Fortunately, however, there are a number of molecular dynamics simulations [28, 29] on the necking deformation of Ni nanowires, which provide some insight on this. Pauly et al. [28] carried out both deformation simulations and conductance calculations on [100]-oriented $\mathrm{Ni}$ nanowires at $4 \mathrm{~K}$ and showed that an atom-sized contact of $\mathrm{Ni}$ takes on distinct stable geometries during atomic scale necking. In the case of noble metals such as $\mathrm{Au}$ or $\mathrm{Ag}$, these stable geometries show different conductance values and produce well-separated peaks in the conductance histogram. By contrast, the conductance of $\mathrm{Ni}$ stable contacts tends to concentrate in a relatively narrow range $(1-1.5) G_{0}$, producing a broad structure in the simulated conductance histogram. This suggests that the $1.3 G_{0}$ peak of Ni may not be a single peak but a composite peak, consisting of contributions from stable contacts slightly larger than a single-atom contact. Calvo et al. [14] closely inspected the $1.3 G_{0}$ peak at $4 \mathrm{~K}$ and found that it is actually a double peak, consisting of two subpeaks at $1.25 G_{0}$ and $1.6 G_{0}$. Though they discuss these subpeaks in terms of spindependent electron transport, the observed double peak structure of the $1.3 G_{0}$ peak strongly suggests that it is composed of multiple contributions. If the 
$1.3 G_{0}$ peak is indeed a composite peak, its disappearance at room temperature would result from thermal broadening. At room temperature, an atom-sized $\mathrm{Ni}$ contact is likely to take more varied geometries than at $4 \mathrm{~K}$ during its necking deformation, as indicated by the nearly smooth conductance trace shown in Fig. 1(c). Because the $3 d$ conductance channels of atom-sized Ni contacts are structure-sensitive, large configurational fluctuations in $\mathrm{Ni}$ contacts at room temperature would result in a wide broadening of their conductance distribution. In the case of $\mathrm{Fe}$, such thermal broadening produces the peak tail but does not wash out the conductance peak. However, for $\mathrm{Ni}$, the broadening of the component contributions would likely smear out the $1.3 G_{0}$ peak, leaving a nearly uniform background. Molecular dynamics simulations of Ni nanowires by Garcia-Mochales et al. [29] find no appreciable broadening of the contact cross-sectional distribution as the temperature rises from $4 \mathrm{~K}$ to room temperature. However, their results are partly inconsistent with those obtained by Pauly et al. [28], probably due to differences in the interatomic potential used in the simulations and the crystallographic orientation of the nanowires. Thus, verifying the composite nature of the $\mathrm{Ni} 1.3 G_{0}$ peak and its thermal smearing at room temperature requires further systematic simulation studies on the necking deformation of Ni nanowires, particularly at room temperature.

\subsection{Comparison with results obtained for solutions}

As mentioned in Sec. 1, Konishi et al. [16] measured the room-temperature conductance histograms of $\mathrm{Fe}, \mathrm{Co}$, and $\mathrm{Ni}$ in electrochemical solutions and reproduced essentially the same conductance histograms as observed at $4 \mathrm{~K}$. However, the authors could observe conductance peaks only under hydrogen evolution conditions, where the dissociated hydrogen adsorbs on the contact surface. Thus, they proposed that atom-sized contacts of $\mathrm{Fe}$, Co, and Ni were stabilized by hydrogen adsorption and became insensitive to large configurational fluctuations at room temperature. Details of such hydrogen-assisted stabilization have been worked out specifically for Ni. [15] In contrast to these contacts in solution, our $\mathrm{Fe}, \mathrm{Co}$, and Ni contacts are in UHV and can bear no protective hydrogen 
layers. Thus, the absence of low-temperature peaks in our room-temperature histograms of $\mathrm{Co}$ and $\mathrm{Ni}$ does not contradict the observation of these peaks in solution, but rather supports the proposed hydrogen-assisted contact stabilization in electrochemical solutions. Presumably, the adsorbed hydrogen stabilizes the contact by lowering its surface energy. This is likely to affect the contact deformation because slip deformation usually produces steps (slip bands) on the surface and increases the surface energy. For atom-sized contacts, this change in surface energy can be significant, causing the contact to follow a few specific deformation paths that involve a smaller number of slip systems. Thus, contact deformation under hydrogen-stabilization conditions becomes essentially similar to deformation at cryogenic temperatures, which would explain why Konishi et al. [16] could reproduce the histograms observed at $4 \mathrm{~K}$.

\section{Conclusion}

We measured the conductance of atom-sized contacts of $\mathrm{Fe}, \mathrm{Co}$, and $\mathrm{Ni}$ in UHV at room temperature and compared the results with those obtained at $4 \mathrm{~K}$. We found that the conductance histograms of $\mathrm{Fe}, \mathrm{Co}$, and Ni showed different responses when the temperature increased from $4 \mathrm{~K}$ to room temperature. For $\mathrm{Fe}$, our conductance histogram nearly reproduced the histogram at $4 \mathrm{~K}$, exhibiting a broad peak around $2.5 G_{0}$. This peak, which was largely unaffected by thermal effects, was probably a single-atom peak of Fe. Our Co and Ni histograms, on the other hand, showed no peak structures, in contrast to their histograms at $4 \mathrm{~K}$. In the case of $\mathrm{Co}$, the histogram was entirely flat, similar to the smooth histograms commonly observed for brittle junctions. As with the similarly flat room-temperature histogram of Ga, the featureless histogram of Co might be the result of the deformation characteristics of atom-sized Co contacts, but the details of this remain unclear. The Ni histogram shows no peaks, but a broad background with an appreciable intensity. We conclude that the conductance peak of Ni observed at $4 \mathrm{~K}$ is a composite peak, consisting of contributions of a number of contact geometries. At room temperature, these component contributions would be thermally broadened to smear out the composite peak into 
a background. The conductance peaks missing in the histograms of Co and $\mathrm{Ni}$ are observed in the case of electrochemical solutions. [16] This corroborates the hydrogen-assisted stabilization of atom-sized $\mathrm{Co}$ and $\mathrm{Ni}$ contacts proposed by Konishi et al.. Without such extrinsic stabilization, the bare atom-sized contacts of $\mathrm{Fe}, \mathrm{Co}$, and $\mathrm{Ni}$ are rather unstable at room temperature, exhibiting short-lived conductance plateaus in conductance traces.

Finally, none of our histograms exhibit integer or half-integer conductance peaks. This clearly demonstrates that the reported fractional peaks are not due to intrinsic properties of atom-sized $\mathrm{Fe}$, Co, and Ni contacts.

\section{References}

[1] N. Agraï, A Levy Yeyati, and J. M. van Ruitenbeek, Phys. Rep. 377 (2003) 81.

[2] K. Oshima and K. Miyano, Appl. Phys. Lett. 73 (1998) 2203.

[3] F. Ott, S. Barberan, J. G. Lunney, J. M. D. Coey, P. Berthet, A. M. de Leon-Guevara, A. Revcolevschi, Phys. Rev. B 58 (1998) 4656.

[4] T. Ono, Y. Ooka, H. Miyajima, and Y. Otani, Appl. Phys. Lett. 75 (1999) 1622.

[5] F. Komori and K. Nakatsuji, J. Phys. Soc. Jpn. 68 (1999) 3786.

[6] F. Elhoussine, S. Mátéfi-Tempfli, A. Encinas, and L. Piraux, Appl. Phys. Lett. 81 (2002) 1681.

[7] J. L. Costa-Krämer, M. Díaz, and P. A. Serena, Rev. Mex. Fis. 47(S1) (2001) 69 .

[8] M. Díaz, J. L. Costa-Krämer, and P. A. Serena, J. Magn. Magn. Mater. 305 (2006) 497.

[9] C. Sirvent, J. G. Rodrigo, S. Vieira, L. Jurkzyszyn, N. Mingo, and F. Flores, Phys. Rev. B 53 (1996) 10686. 
[10] J. L. Costa-Krämer, Phys. Rev. B 55 (1997) R4875.

[11] K. Hansen, E. Lægsgaard, I. Stensgaard, and F. Besenbacher, Phys. Rev. B 56 (1997) 2208.

[12] B. Ludoph and J. M. van Ruitenbeek, Phys. Rev. B 61 (2000) 2273.

[13] C. Untiedt, D. M. T. Dekker, D. Djukic, and J. M. van Ruitenbeek, Phys. Rev. B 69 (2004) 081401(R).

[14] M. R. Calvo, M. J. Caturla, D. Jacob, C. Untiedt, and J. J. Palacios, IEEE Trans. Nanotech. 7 (2008) 165.

[15] M. Kiguchi, T. Konishi, and K. Murakoshi, Appl. Phys. Lett. 87 (2005) 043104.

[16] T. Konishi, M. Kiguchi, and K. Murakoshi, Surf. Sci. 602 (2008) 2333.

[17] M. R. Calvo, J. Fernández-Rossier, J. J. Palacios, D. Jacob, D. Natelson, and C. Untiedt, Nature 458 (2009)1150.

[18] T. Nakazumi and M. Kiguchi, J. Phys. Chem. Lett. 1 (2010) 923.

[19] H. Egle, C. Bacca, H.-F. Pernau, M. Huefner, D. Hinzke, U. Nowak, and E. Scheer, Phys. Rev. B 81 (2010) 134402.

[20] Recent atom-contact experiment at $4 \mathrm{~K}$ using cryogenic STM (N. Néel et al., Phys. Rev. Lett. 102 (2009) 086805) observes a $G_{0} / 2$ conductance for a $\mathrm{Ni}$ tip touching with a single Co adatom on a Co island, suggesting that the realization of the half-integer quantized conductance critically depends on the contact geometry.

[21] D. Miura, K. Iwata, S. Kurokawa, and A. Sakai, e-J. Surf. Sci. Nanotech. 7 (2009) 891.

[22] M. Tsutsui, K. Shoji, M. Taniguchi and T. Kawai, Nano Lett. 8 (2008) 345.

[23] E. Scheer, P. Konrad, C. Bacca, A. Mayer-Gindner, H. V. Löhneysen, M. Häfner, and J. C. Cuevas, Phys. Rev. B 74 (2006) 205430. 
[24] R. Suzuki, Y. Mukai, M. Tsutsui, S. Kurokawa, and A. Sakai, Jpn. J. Appl. Phys. 45 (2006) 7217.

[25] R. H. M. Smit, A. I. Mares, M. Häfner, P. Pou, J. C. Cuevas, and J. M. van Ruitenbeek, New J. Phys. 11 (2009) 073043.

[26] B. B. Lewis, K. G. Vandervoort, and R. D. Foster, Solid State Commun. 109 (1999) 525.

[27] A. Takahashi, M. Mabuchi, S. Kurokawa, and A. Sakai, Unpublished.

[28] F. Pauly, M. Dreher, J. K. Viljas, M. Häfner, J. C. Cuevas, and P. Nielaba, Phys. Rev. B 74 (2006) 235106.

[29] P. García-mochales, S. Peláez, P. A. Serena, E. Medina, and A. Hasmy, Appl. Phys. A 81 (2005) 1545. 

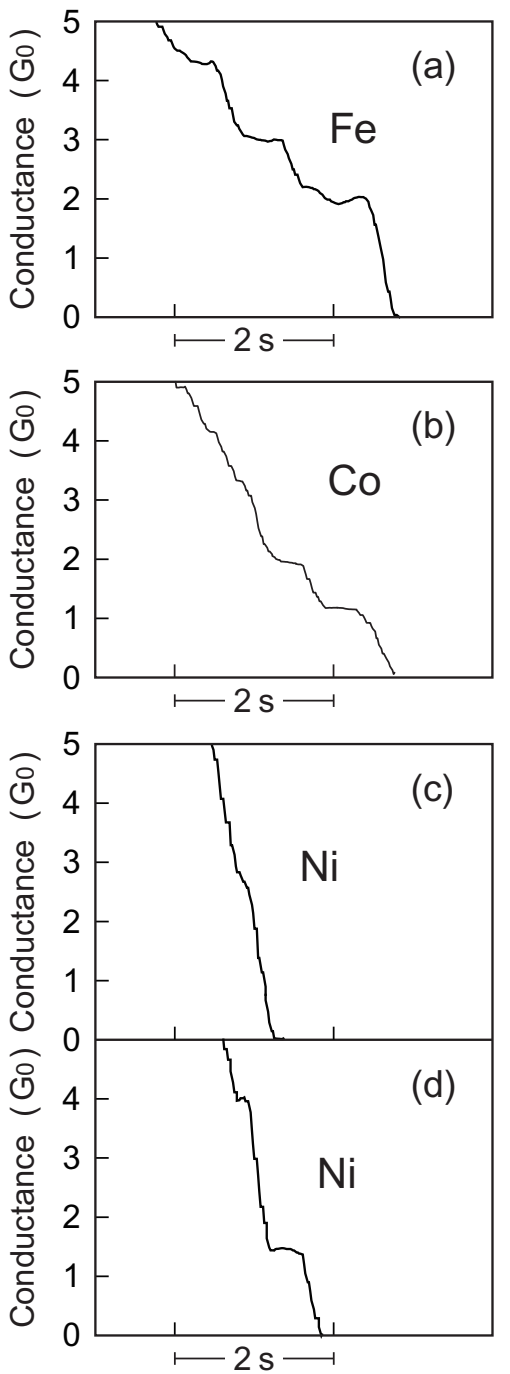

Figure 1: Typical conductance traces of (a) Fe, (b) Co, and (c)-(d) Ni atom-sized contacts. The two-second bar in the time scale would correspond to an apparent electrode displacement of $\sim 1.4 \mathrm{pm}$ if we use our opening speed estimate of $\sim 0.7 \mathrm{pm} / \mathrm{s}$. Under such a slow opening speed, junctions are considered to undergo self-breaking [22], as mentioned in Sec. 3.1. 

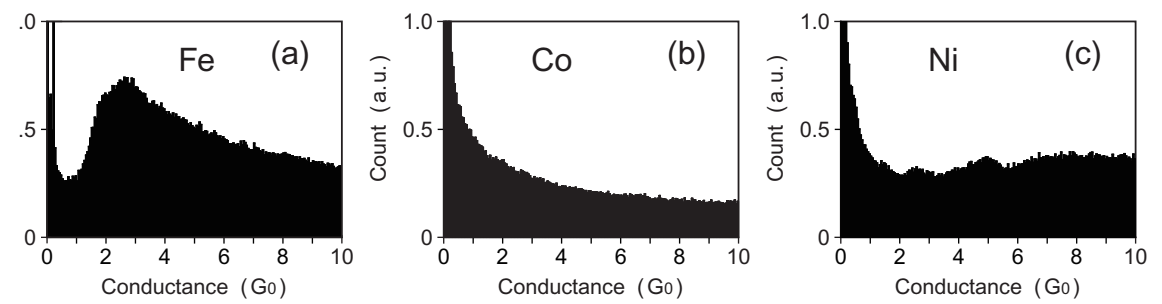

Figure 2: Conductance histograms of (a) Fe, (b) Co, and (c) Ni obtained at room temperature in UHV. 